\title{
A Modeling Approach to Fiber Fracture in Melt Impregnation
}

\author{
Feng Ren ${ }^{1,3}$, Cong Zhang ${ }^{1}$, Yang $\mathrm{Yu}^{2}$, Chunling $\mathrm{Xin}^{1}$, Ke Tang ${ }^{1}$ and Yadong $\mathrm{He}^{1,3 *}$ \\ ${ }^{1}$ College of Mechanical and Electrical Engineering, Beijing University of Chemical Technology, Beijing 100029, \\ China \\ ${ }^{2}$ School of Civil and Environmental Engineering, University of Technology Sydney, NSW 2007, Australia \\ ${ }^{3}$ Polymer Processing Equipment Engineering Research Centre, Ministry of Education, Beijing 100029, China \\ *Corresponding author: heyd@ mail.buct.edu.cn
}

\begin{abstract}
The effect of process variables such as roving pulling speed, melt temperature and number of pins on the fiber fracture during the processing of thermoplastic based composites was investigated in this study. The melt impregnation was used in this process of continuous glass fiber reinforced thermoplastic composites. Previous investigators have suggested a variety of models for melt impregnation, while comparatively little effort has been spent on modeling the fiber fracture caused by the viscous resin. Herein, a mathematical model was developed for impregnation process to predict the fiber fracture rate and describe the experimental results with the Weibull intensity distribution function. The optimal parameters of this process were obtained by orthogonal experiment. The results suggest that the fiber fracture is caused by viscous shear stress on fiber bundle in melt impregnation mold when pulling the fiber bundle.
\end{abstract}

Keywords: fiber fracture mechanism, melt impregnation, Weibull distribution, continuous fibers, orthogonal experiment

\section{Introduction}

Continuous fiber reinforced thermoplastic composite materials have been widely used in many fields of aerospace, automobile, chemical and electronic appliances, etc [1-5]. These materials can be processed by numerous techniques including filament winding, pultrusion and prepreg. The application of prepreg-related products is the mainstream technology on the current market [6,7]. The melt impregnation process has been the main technology in the industry to produce the prepreg due to its simplicity relatively $[8,9]$.

With the wide application of the composite material, more and more people place emphasis on the research of raw materials, composite technology, interface theory, composite effect and theory, and have made great achievements [10]. But study on the fracture of fibers during the composite process just stay at the empirical level and the effect of the experimental process parameters and the raw material structure parameters on fiber fracture during impregnation process cannot be accurately described.

With the process of melt impregnation in this paper, the 2400tex glass fiber was impregnated in the molten poor, which was designed in the laboratory, to get the prepreg. A fiber bundle fracture model related to process parameters was established through the research on influence of resin on fiber bundle breakage on the base of Weibull intensity distribution function [11].

\section{Model Development}

\subsection{Previous Work}


In the final analysis, the breakage of brittle material is caused by the stress on fiber. The force on fiber during impregnation must be made clear to study the mechanism of fracture in the process. Turkovich et al. studied the fiber fracture at different location of single screw extruder [12]. His analysis of how fibers break during processing suggests that there are three areas causing fiber damage including fiber/fiber interaction, fiber contact with processor surfaces and fiber interaction with the polymer. Most of the damage occurred in the screw melting zone, although when fibers are added after the melting zone, sufficient shear stress occurs in the mixing zone to break fibers. Astrom and Pipes presented a model to calculate the accumulated pulling resistance from a pultrusion die, arising from viscous, compaction, and friction resistance [13,14]. The model was evaluated studying an ideal pultrusion process for manufacturing of unidirectional fiber-reinforced thermoplastic. According to their model, it may be concluded that the pulling force is slightly less than directly proportional to the pulling speed. With a Newtonian fluid model the pulling force is approximately proportional to the pulling speed, but the shear thinning of the matrix lessens this dependence. Yun and Lee analyzed pultrusion process of phenolic foam composite and carried out a model to predict the pulling force during the process [15]. In their studies, the puling force applied on fiber-reinforced foam composite is consist of shearing force, which is caused by viscous shear between the die wall and fiber bundle, and the Coulomb friction between the composite and the die wall. Srinivasagupta et al. developed a pull-force model based on the assumption that the resin back-flow and the viscous drag of the part on the die wall are the contributing factors [16]. As shown in Fig. 1, the schematic of the pull-force model can be divided into two sections. In the heating section, the major contribution to the pull force is the viscous drag arising when a thin layer of resin is sheared between the fibers and the die wall. The evolution of the fiber-length distribution along a twin-screw extruder was studied and modeled by Durin et al. [17]. Their results indicated that the fiber systematically breaks-up because of the resulting huge deformation when buckling occurs. R'Mili et al. used the Weibull intensity distribution function to explain fracture of brittle materials such as glass fiber [18]. They found out ttwo Weibull parameters through the tension tests on loose bundles.

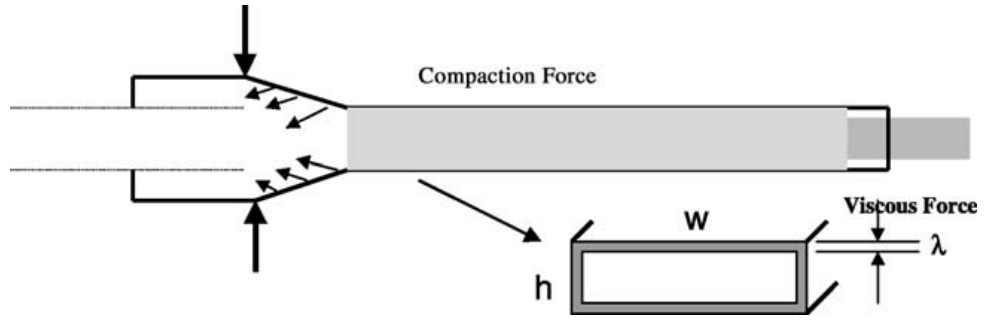

Fig.1 Schematic of the stress on fiber

Therefore, we can set some conditions to establish the stress model on fibers during the melt impregnation process and predict the fiber fracture combined with the Weibull distribution.

\subsection{Stress Model}

The process of resin impregnating continuous fiber in the molten poor can be simplified to fiber bundle towed in resin melt. We make two assumptions as follows:

(1)The fiber bundle is considered to be an elliptic cylinder which contains thousands of monofilaments. The monofilaments inside the bundle are evenly distributed and deemed to be paralleled to each other.

(2)The resin inside the fiber bundle has the same velocity as the monofilaments. The effect of the contact stress among the monofilaments and the gravitational influence in the fluid flow is ignored

A physical model is established on the basis of the two assumptions above in cylindrical coordinates and is shown in Fig. 2. The tension equilibrium equation of fiber infinitesimal can be written: 


$$
F_{\mu}=F(z+d z)-F(z)
$$

where $F_{\mu}$ is the force caused by the effect of resin the viscous resistance, $F(z+d z)$ and $F(z)$ the tension on both ends of infinitesimal.

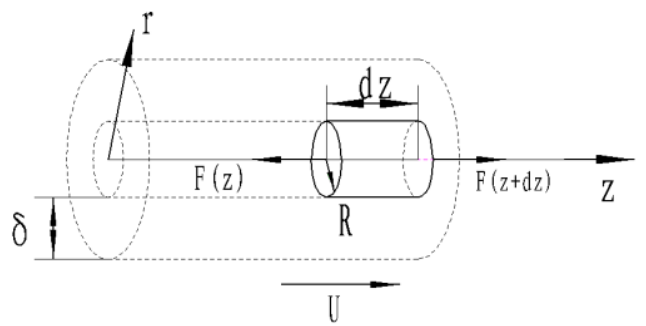

Fig. 2 Tension on the fiber bundle in cylindrical coordinates

The shear stress in the molten poor is calculated according to the continuity equation and momentum equation in hydrodynamic. The velocity field around the fiber bundle is calculated by solving the continuity equation and momentum equation:

$$
u_{z}=\frac{U}{\ln \frac{R}{R+\delta}}[\ln R-\ln (R+\delta)]
$$

where $U$ is the pulling speed, $R$ is the equivalent radius, and $\delta$ is the thickness of boundary lubrication layer. Then we can obtain the shear stress on fiber bundle:

$$
\tau_{z r_{r=R}}=\mu\left(\frac{\partial u_{z}}{\partial r}+\frac{\partial u_{r}}{\partial z}\right)=\mu \frac{U}{R \ln \frac{R}{R+\delta}}
$$

Then the force and stress caused by shear drag on fiber bundle are equation (4) and (5):

$$
\begin{gathered}
\mathrm{F}_{\mu}=\tau_{\mathrm{zr}_{\mathrm{r}=\mathrm{R}}} \times 2 \pi \mathrm{Rdz}=\frac{2 \pi \mu \mathrm{Udz}}{\ln \frac{\mathrm{R}}{\mathrm{R}+\delta}} \\
\sigma=\frac{\mathrm{F}_{\mu}}{\mathrm{s}}=\mu \frac{2 \mathrm{Udz}}{\mathrm{NR}_{\mathrm{f}}^{2} \mathrm{Mln} \frac{\mathrm{R}}{\mathrm{R}+\delta}}
\end{gathered}
$$

where $\mu$ is the viscosity of resin, $R_{f}$ is the radius of fiber, $N$ is the number of filaments and $M$ is the degree of impregnation. The degree of impregnation, $M$, can be estimated by equation (6):

$$
\mathrm{M}=1-\emptyset
$$

where $\phi$ is the porosity. From equation (4) and equation (1), the following equation was obtained by integral method:

$$
\sigma(\mathrm{l})=\mu \frac{2 \mathrm{Ul}}{\mathrm{NR}_{\mathrm{f}}^{2} \mathrm{Mln} \frac{\mathrm{R}}{\mathrm{R}+\delta}}
$$

where $l$ is the distance to die entrance. The viscosity of thermoplastic resin depends on the local shear rate and temperature. From this we can know the influence of temperature on the fiber bundle fracture rate is mainly reflected in the viscosity. Under a certain shear rate, temperature dependence of the polymer melt viscosity can be described by the Arrhenius equation :

$$
\mu=\mu_{\infty} \exp \left(\frac{\mathrm{E}_{\dot{\gamma}}}{\mathrm{RT}}+\mathrm{k} \alpha\right)
$$

where $\mu_{\infty}, \mathrm{E}_{\dot{\gamma}}$ and $\mathrm{k}$ are the are empirically determined constants for a given resin under a certain shear rate, $\mathrm{R}$ 
is the ideal gas constant, $\mathrm{T}$ is the thermodynamic temperature, and $\alpha$ is the degree of cure (the value in this paper is 0 ).

The influence caused by pin on fiber bundle includes two aspects.

(1) The viscous shear stresses between the roving and the pin occur in a region of constant gap thickness located between the theoretical points of tangency of the roving and the pin. This region is shown schematically in Fig. 3 . We can obtain the equation (9) by the same way above:

$$
\sigma_{\mathrm{f}}=\mu \frac{2 \mathrm{US}}{\mathrm{NR}_{\mathrm{f}}^{2} \mathrm{Mln} \frac{\mathrm{R}}{\mathrm{R}+\varepsilon}}
$$

where $S$ is the contact length, and $\varepsilon$ is the gap thickness. The gap thickness can be determined by equation (10):

$$
\varepsilon=\mathrm{R}_{\mathrm{f}}\left(1-0.5 \sqrt{\frac{\sqrt{3} \pi \mathrm{V}_{\mathrm{f}}}{2}}\right)
$$

where $v_{f}$ is the fiber content.

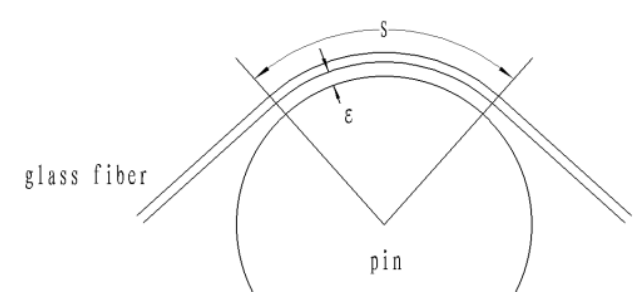

Fig. 3 Gap between fiber and pin

(2) The Bending stress: breakage occurs because of the severe tensile stresses induced on the external surface of the fibre when it is bending. This stress depends on the fiber radius $R_{f}$, its Young modulus $E$ and the pin diameter $D$ :

$$
\sigma_{\mathrm{b}}=\frac{2 \mathrm{ER}_{\mathrm{f}}}{\mathrm{D}}
$$

Equations (6) to (11) can be combined to yield the following differential equation for stress on fiber:

$$
\sigma_{\max }=\sigma\left(l_{0}\right)+\sigma_{\mathrm{b}}+n \sigma_{\mathrm{f}}
$$

where $\mathrm{n}$ is the number of pins, and $l_{0}$ is the distance between the furthest pin and die entrance. As a kind of brittle material, the breaking strength of glass fiber can be described by Weibull distribution:

$$
\mathrm{P}=1-\mathrm{e}^{-\left(\frac{\sigma_{\max }}{\sigma_{0}}\right)^{\mathrm{m}}}
$$

where $P$ is the fracture rate of fiber bundle, $\sigma_{0}$ is the scale parameter, and $m$ is the shape parameter. Combining equations (11) and (12) leads to the relationship between fiber fracture and process parameters. It is obvious that the pin number, pulling speed and processing temperature have a great influence on fiber fracture.

\section{Experimental Melt Impregnation Procedure and Material Variables}

\subsection{Experimental Procedure}

The melt impregnation process used in this research is shown schematically in Fig. 4. The glass roving was unwound by dispersion rollers, preheated by preheating unit and pulled over staggered cylindrical pins located in an atmospheric pressure, self-designed melt pool. The roving, impregnated with melt, was pulled from the pool through an outlet die by a double belt puller. 


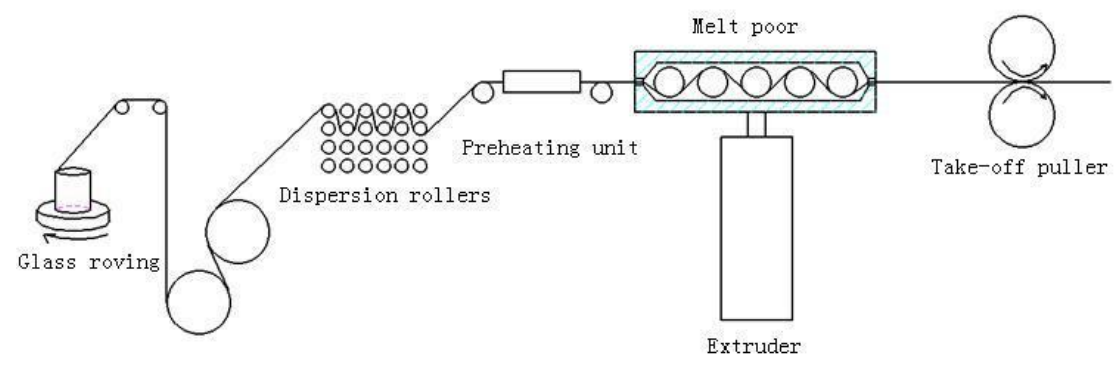

Fig. 4 Schematic drawing of the melt impregnation process used

The fracture rate of fiber bundle and the porosity of prepreg were measured under the standard GB/T 7690.1-2001, GB/T 2577-2005 and GB/T 1463-2005 with the method of calcination using Muffle: SX2-4-10, Wuhan Yahua Electric Stove Co., LTD. Fracture rate means the ratio of mass loss per unit length of the fiber bundle after impregnation to original fiber bundle, which is called the loss of line density. Porosity means the proportion of pores in the material, which directly reflects the quality of impregnation of the prepreg. Here we use the density method to calculate the composite apparent porosity, as the following equations:

$$
\begin{gathered}
\emptyset=\frac{100\left(\mathrm{~T}_{\mathrm{D}}-\mathrm{M}_{\mathrm{D}}\right)}{\mathrm{M}_{\mathrm{D}}} \\
\mathrm{M}_{\mathrm{D}}=\frac{\mathrm{m}}{\mathrm{v}} \\
\mathrm{T}_{\mathrm{D}}=\frac{\mathrm{m}}{\mathrm{m}_{\mathrm{f}} / \rho_{\mathrm{f}}+\mathrm{m}_{\mathrm{pp}} / \rho_{\mathrm{pp}}}
\end{gathered}
$$

where $M_{d}$ is the measured density, $T_{d}$ is the theoretical density, $m$ is the total mass of sample, $m_{f}$ is the fiber mass, $\rho_{f}$ is the fiber density, $m_{p p}$ is the polypropylene(PP) mass, and $\rho_{p p}$ is the $\mathrm{PP}$ density.

\subsection{Equipment Operating Parameters}

Constant temperature polymer melt was fed to the pool using a single screw extruder-Brabender. The melt pool temperature was held constant using 6 independently controlled band heaters. The roving pulling speed $(U)$ could be varied between 30 and $50 \mathrm{~mm} / \mathrm{s}$.

\subsection{Material Parameters}

The thermoplastic polymer used in this melt impregnation study was polypropylene from SK energy: BX3900. Rheological parameters of the polymer can be measured by a capillary rheometer- Thermo Fisher Scientific (China) Co., LTD. The glass roving used in this research was a 2400 tex direct wound roving product developed for melt impregnation compounding processes by Jushi Group LTD. The roving is composed of 4400 sized glass monofilaments of diameter $17 \mu \mathrm{m}$. The shape parameters and the scale parameters can be obtained through the fiber bundle tensile tests [15] using the computer-controlled electronic universal testing machine: RGM-100A, Shenzhen Reger instrument Co., LTD. The results are given as follows: $m=2.61795, \sigma_{0}=820.25 \mathrm{Mpa}$.

\section{Results and Discussion}

\subsection{Effect of Pulling Speed}


Since pultrusion is an automatic and continuous process for fabricating fiber reinforced plastics, the proper pulling speed is a critical parameter in the optimization of the production rate and the mechanical properties of the composites $[19,20]$. Fig. 5 illustrates the effects of pulling speed on the degree of impregnation. It is known that the degree of impregnation decreases when pulling speed increases. The reason is that the increasing pulling speed leads to a decreasing residence time for fiber bundle in the mold. This means the resin melt does not have enough time to penetrate into the fiber bundle and then the fiber bundle is pulled out.

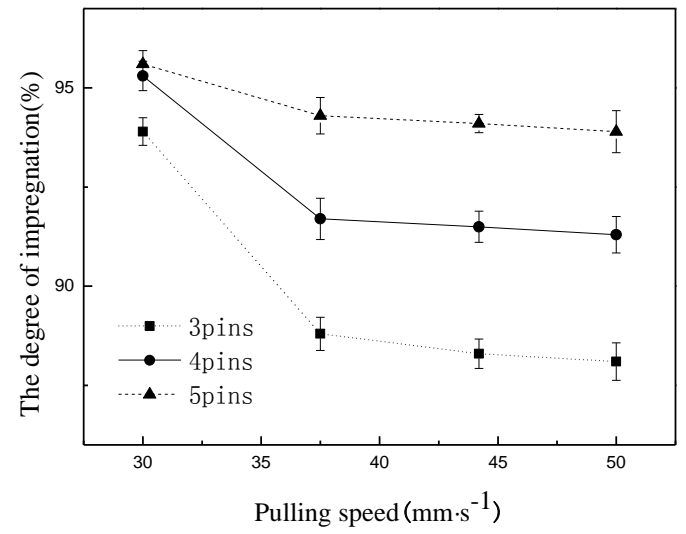

Fig. 5 Effects of pulling speed on the degree of prepreg impregnation at $493 \mathrm{~K}$

Fig. 6 shows the fracture rate as a function of pulling speed for 3 and 5 pins of diameter $20 \mathrm{~mm}$. With the increase of the pulling speed, the fiber fracture rate gradually increased. The higher pulling speed, the higher shear rate will be. The higher shear rate causes the higher shear stress. And this increases the fracture rate of fiber bundle. Close agreement between the two results is observed. The results confirmed the fracture of fiber bundle model. People often improve the pulling speed for the pursuit of productivity and this not only leads to the reduction of impregnation degree but also makes the increasing fracture rate of fiber bundle until the fiber bundle the emergence of fiber bundle break down. This influences the performance of the product and process stability seriously.

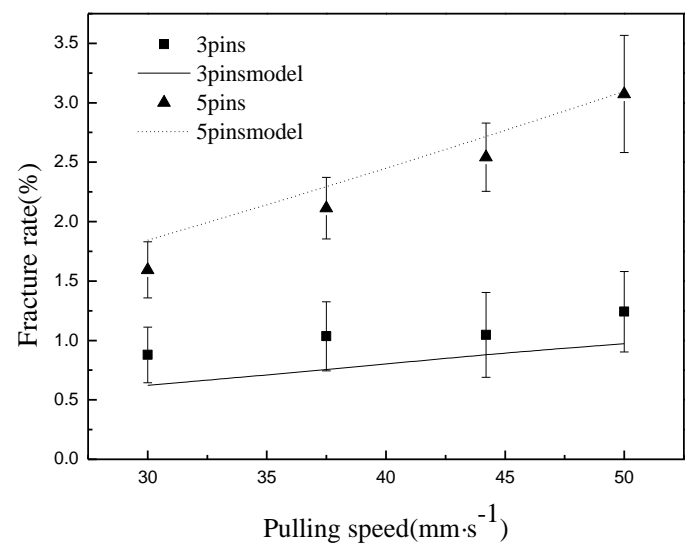

Fig. 6 Fracture rate as a function of puling speed for three and five pins of diameter $20 \mathrm{~mm}$ at $493 \mathrm{~K}$

\subsection{Effect of Pin Number}

The degree of impregnation with different pin numbers is measured (see Fig.7). In this process, the roving is pulled over a pin and, in between the roving and the pin, a melt film is present. The fiber bundle is pulled into the polymer film or that the melt film, which is under pressure, diffuses into the fiber [21]. The more pins, the more times fiber bundle goes through this wedge zone with pressure. Because of that, more pins are usually used 
in order to improve the degree of impregnation. But this effect is limited when the degree of impregnation is over $95 \%$.

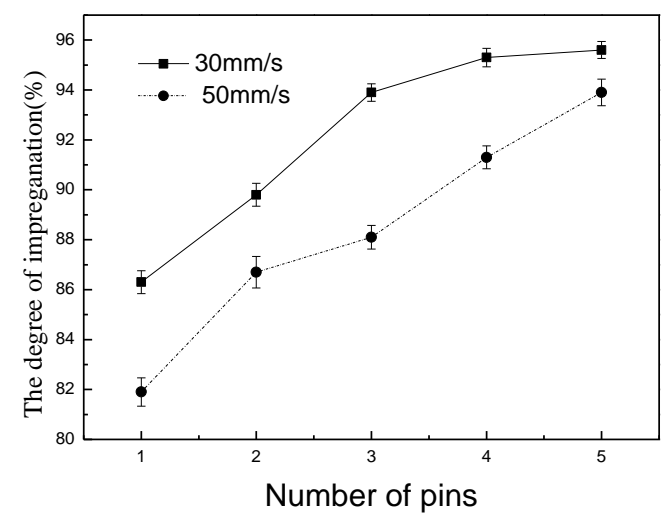

Fig. 7 Effect of the number of the pin on the degree of prepreg impregnation at $493 \mathrm{~K}$

Fig. 8 is the relationship between the fracture rate of fiber bundle and the number of pins. It is visible that fracture rate is observed to increase with increasing pin numbers. The pin-roving contact angle increases when more pins are used. When fiber bundle goes through the pins from the first one to the last one, this increases the contact length in small gap shear field, in where fiber bundle is inflicted serious shear stress. And then the fracture rate of fiber bundle increases just as the Weibull distribution function described.

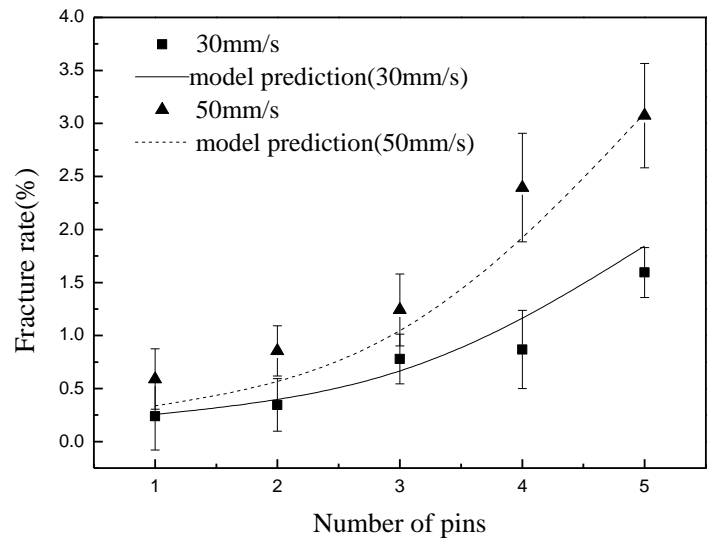

Fig. 8 Effect of the pin number on fracture rate of fiber at $493 \mathrm{~K}$.

\subsection{Effect of Processing Temperature}

Different pulling speeds correspond to different shear rates which influence the flow activation energy. First, the rheological properties of polypropylene in this study are measured under different temperatures. Then, the viscosity-shear rate relationships are obtained from supplier data by fitting in the model of power-law fluid as shown in Fig. 9 (a). Finally, the viscosity-temperature relationships at a certain shear rate are obtained from the calculated viscosity at different temperatures as shown in Fig. 9 (b). The lower shear rate and temperature, the higher viscosity resin will be and the resin used in this study is temperature-insensitive.

Fig. 10 shows the degree of impregnation at different processing temperature. Resin viscosity decreases with increasing temperature and resin can more easily penetrate into the fiber bundle. So the degree of impregnation increases. An increase in processing temperature has limited positive effect on the degree of impregnation especially when the temperature is above 493K. Fig. 11 illustrates fracture rate as a function of processing temperature. Theoretical and experimental data figure out that an increase processing temperature can lower the fracture of fiber bundle. The decreasing viscosity means that the resin dragging effect is weak, the shear stress on 
fiber is small, so the decreasing fracture rate.
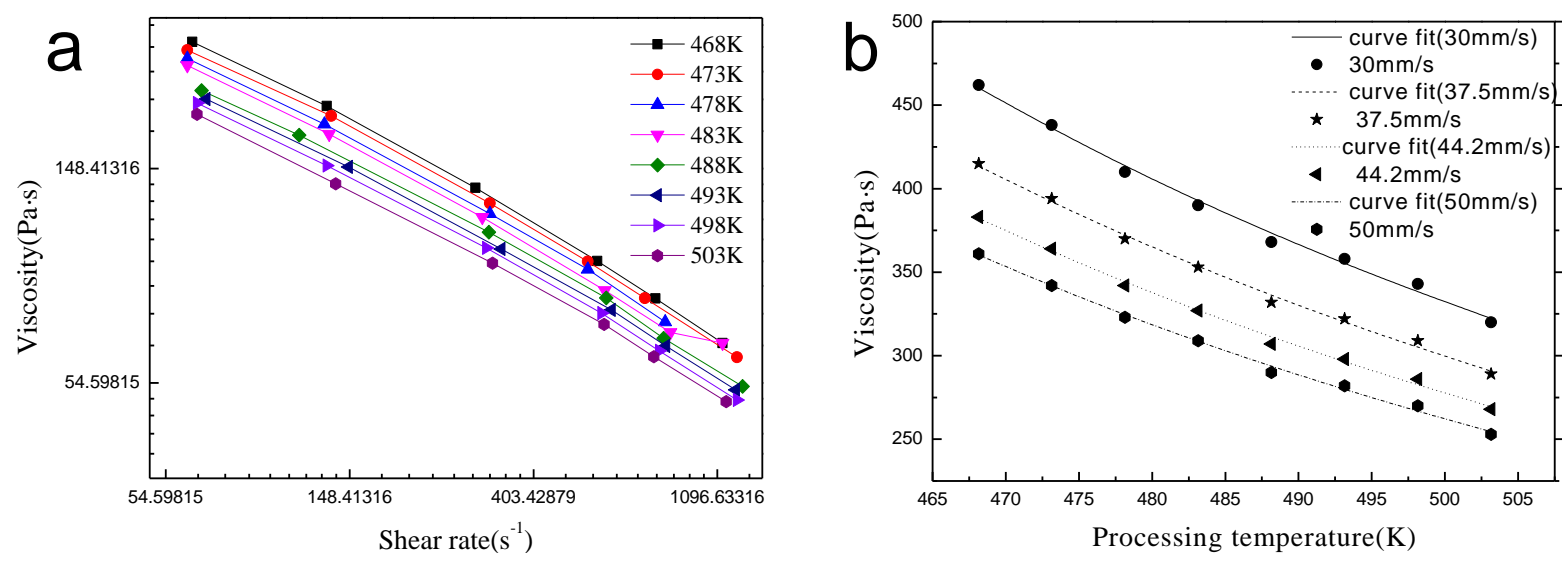

Fig. 9 Relationships between viscosity and shear rate: a viscosity of resin at different temperature, and $\mathbf{b}$ effects of temperature on viscosity of resin at different shear rates.

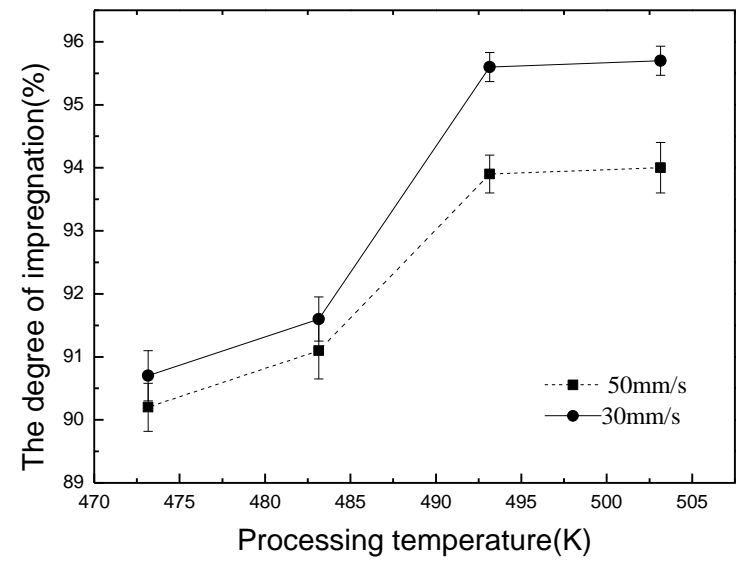

Fig. 10 Effects of processing temperature on the degree of prepreg impregnation for five pins

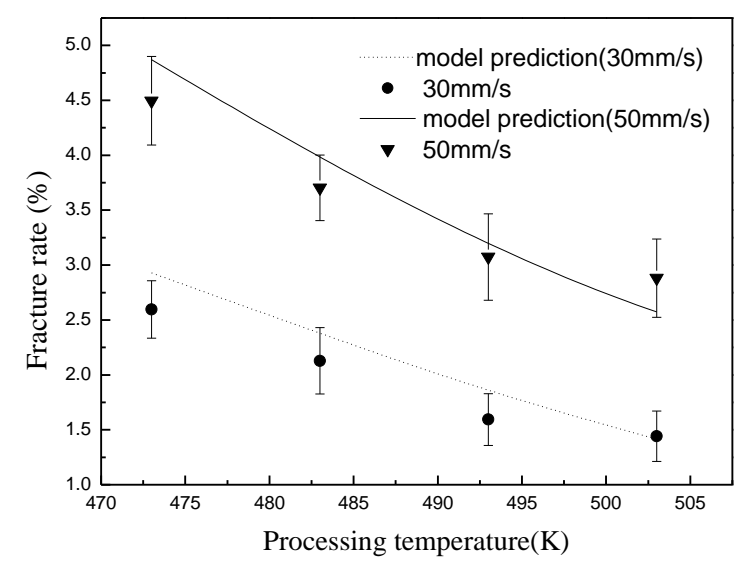

Fig. 11 Fracture rate as a function of processing temperature for five pins

\subsection{Model Discussion}

There are three processing parameters having the effect on the degree of impregnation and the fracture rate of fiber bundle in this model, such as the processing temperature, pin number and pulling speed. In this study, orthogonal design analysis method was used to investigate the effects of the three factors. For each factor, four 
levels were set respectively as illustrated in table 1 and 2 .

Table 1 Factor level

\begin{tabular}{cccc}
\hline Level & A: processing temperature $(\mathrm{K})$ & B: pin number & C: pulling speed $(\mathrm{mm} / \mathrm{s})$ \\
\hline 1 & 473 & 2 & 30 \\
2 & 483 & 3 & 37.5 \\
3 & 493 & 4 & 44.2 \\
4 & 503 & 5 & 50 \\
\hline
\end{tabular}

Table 2 Orthogonal test schedule

\begin{tabular}{cccc}
\hline No. & A: processing temperature $(\mathrm{K})$ & B: pin number & C: pulling speed $(\mathrm{mm} / \mathrm{s})$ \\
\hline 1 & 473 & 5 & 44.2 \\
2 & 473 & 4 & 30 \\
3 & 473 & 2 & 37.5 \\
4 & 473 & 3 & 50 \\
5 & 483 & 2 & 44.2 \\
6 & 483 & 4 & 37.5 \\
7 & 483 & 3 & 30 \\
8 & 483 & 5 & 50 \\
9 & 493 & 4 & 44.2 \\
10 & 493 & 3 & 37.5 \\
11 & 493 & 5 & 30 \\
12 & 493 & 2 & 50 \\
13 & 503 & 4 & 50 \\
14 & 503 & 5 & 37.5 \\
15 & 503 & 2 & 30 \\
16 & 503 & 3 & 44.2 \\
\hline
\end{tabular}

$\mathrm{R}$, the difference between the maximum and the minimum of ki, was used to express the influence grade of factors. ki was the average of the three results in level $\mathrm{i}$ under one factor. The larger $\mathrm{R}$, the greater impact of corresponding factor had on the response variable. So the selected factor with the largest $\mathrm{R}$ is the most important one. The responses of fracture rate and the degree of impregnation are showed in table 3 .

Table 3 Fracture rate of fiber bundle and the degree of impregnation

\begin{tabular}{ccc}
\hline No. & The degree of impregnation $(\%)$ & Fracture rate of fiber bundle $(\%)$ \\
\hline 1 & 90.35 & 4.315 \\
2 & 90.5 & 1.720 \\
3 & 84.24 & 0.578 \\
4 & 87.3 & 1.356 \\
5 & 85.28 & 0.557 \\
6 & 91 & 1.740 \\
7 & 90.1 & 0.753 \\
8 & 91.1 & 3.956 \\
9 & 92.46 & 1.660 \\
10 & 91.73 & 0.749 \\
\hline
\end{tabular}




\begin{tabular}{lcc}
\hline 11 & 95.6 & 1.843 \\
12 & 86.7 & 0.524 \\
13 & 91.4 & 1.589 \\
14 & 95.06 & 1.929 \\
15 & 90.2 & 0.339 \\
16 & 89.98 & 0.770 \\
\hline
\end{tabular}

Fig. 12 and Fig. 13 show that ki of the degree of impregnation and fracture rate under different processing conditions. It could be seen that the number of pins had the greatest impact on degree of impregnation and fracture rate of fiber bundle. The number of pins acts an important role on impregnation, as well as fiber breakage, during the processing. An increasing pin number not only has limited positive influence on impregnation when the degree of imprenation is above $94 \%$ but also causes a seriously increasing fracture rate of fiber bundle. When the pin number is over eight, the puller cannot pull out the prepreg at all.

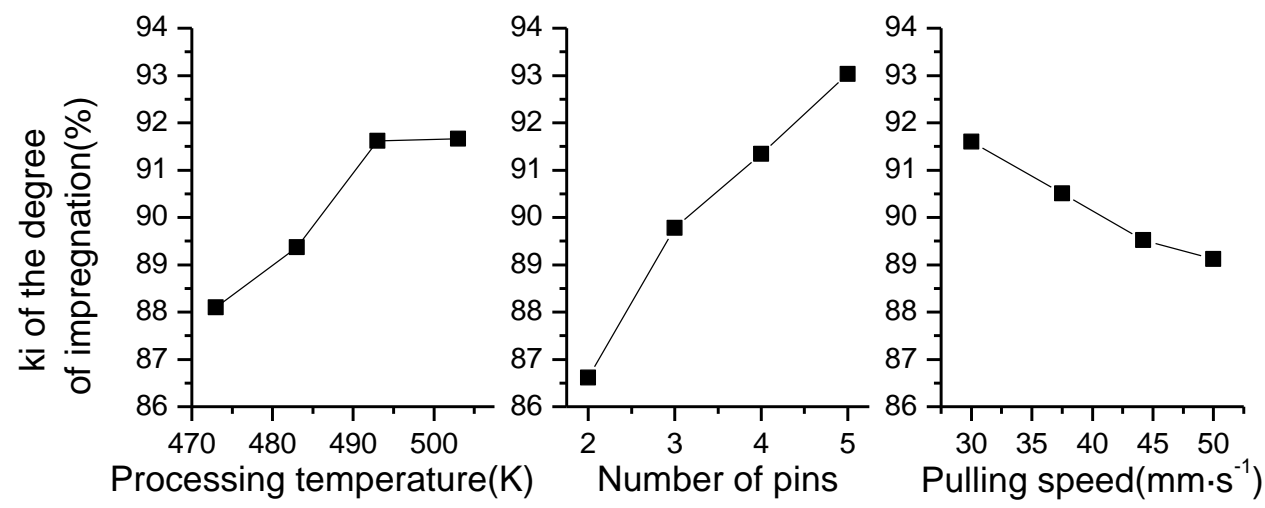

Fig. 12 Effect of four factors on the degree of impregnation

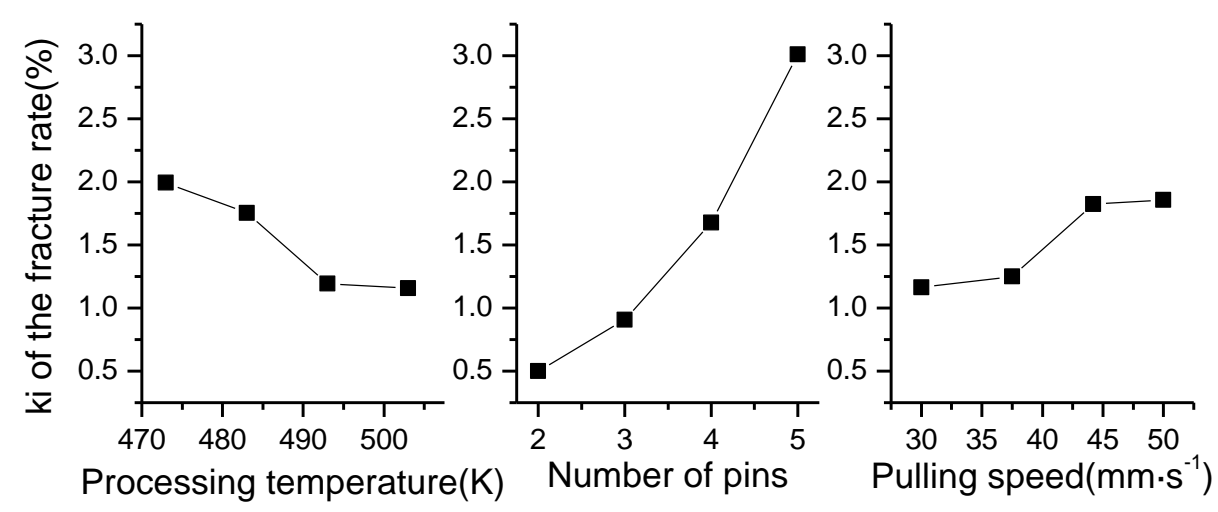

Fig. 13 Effect of three factors on the fracture rate

Fig. 14 and Fig. 15 show the degree of impregnation and fracture rate of fiber bundle as a function of puling speed and the pin number with DOE software simulation at two different processing temperatures. Through the 3D graphics of the simulation function, we can directly determine the optimal processing parameters to ensure the maximum yield (equivalent to the maximum towing speed) of the prepreg in the condition of more than $95 \%$ in the impregnation degree and less than 5\% fracture rate. The optimal processing parameters are given as follows: The pulling speed is $37.5 \mathrm{~mm} / \mathrm{s}$ for five pins at $503 \mathrm{~K}$. 

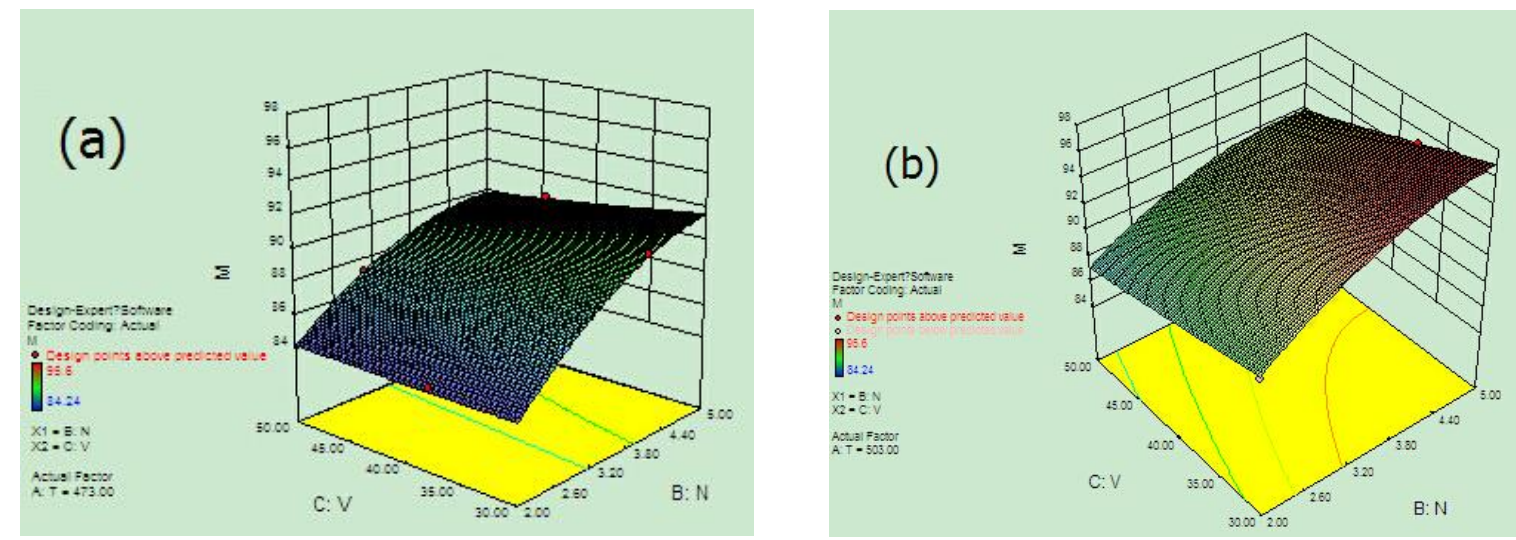

Fig. 14 The degree of impregnation as a function of puling speed and the pin number with DOE software simulation at: a $473 \mathrm{~K}$, and b $500 \mathrm{~K}$
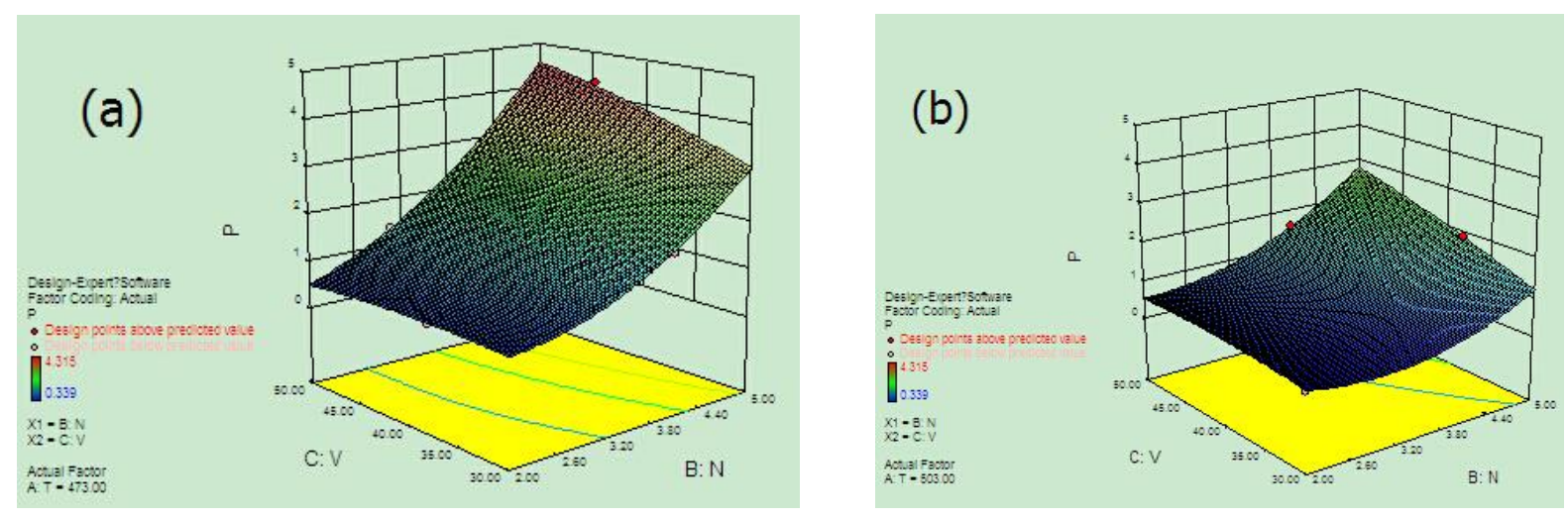

Fig. 15 The fracture rate of fiber bundle as a function of puling speed and the pin number with DOE software simulation at: a $473 \mathrm{~K}$, and b $500 \mathrm{~K}$

\section{Conclusions}

(1) A mathematical model of fiber fracture during processing of thermoplastic based composites was established with the application of Weibull intensity distribution theory to describe the relationships of continuous fiber breakage and the experimental process conditions, as well as material parameters (resin viscosity and glass fiber bundle structure parameters) in the impregnation process. The reliability of the model was verified by experiments.

(2) The number of pins, which is the most significant factor in this process, has little impact on impregnation when the degree of impregnation is over $95 \%$. When the processing temperature is higher than $493 \mathrm{k}$, it has little impact on impregnation and fiber fracture.

(3) We can decrease the fiber fracture by using few pins in the precondition of satisfying a certain degree of impregnation. If we want to get prepreg with high quality, we should use a low pulling speed, the appropriate processing temperature and pin numbers. But the pin number cannot be more than 7 to ensure that the prepreg can be pulled out continuously.

\section{Acknowledgement}

This work was supported by the National Natural Science Foundation of China (Grant No. 51273019). The 
authors are also thankful for the beneficial suggestions and comments from the editor and reviewers.

\section{References}

1. Xing, L., Jiang, S., Zhou, Z.: Progress of manufacturing technology development of advanced polymer matrix composites. Acta Materiae Compositae Sinica 30(2), 1-9 (2013)

2. Bohm, R., Hufnagl, E., Kupfer, R., Engler, T., Hausding, J. Cherif, C., Hufenbach, W.: Thermoplastic composites reinforced with textile grids: development of a manufacturing chain and experimental characterization. Appl. Compos. Mater. 20(6), 1077-1096 (2013)

3. Bozic, M., Fleischhauer, R., Kaliske, M.: Thermomechanical modeling of fiber reinforced material including interphasial properties and its application to epoxy/glass. Eng. Computation. 33(4), 1259-1281 (2016)

4. Risicato, J., Kelly, F., Soulat, D., Legrand, X., Trumper, W., Cochrane, C., Koncar, V.: A complex shaped reinforced thermoplastic composite part made of commingled yarns with integrated sensor. Appl. Compos. Mater. 22(1), 81-98 (2015)

5. Marissen, R., Drift, L.T.V.D., Sterk, J.C.: Folding of continous fibre thermoplastic composites. Appl. Compos. Mater. 4(5), 273-282 (1997)

6. Vallittu, P.K.: Impregnation of glass fibres with polymethylmethacrylate using a power-coating method. Appl. Compos. Mater. 2(1), 51-58 (1995)

7. Haffner, S.M., Friedrich, K., Hogg, P.J., Busfield J.J.C.: Finite element assisted modelling of the microscopic impregnation process in thermoplastic preforms. Appl. Compos. Mater. 5(4), 237-255 (1998)

8. Steggall-Murphy, C., Simacke, P., Advani, S.G., Yarlagdda, S., Walsh, S. A model for thermoplastic melt impregnation of fiber bundles during consolidation of power-impregnated continuous fiber composites. Part A-Appl. S. 41(1), 93- 100 (2010)

9. Xian, G., Pu, H.T., Yi, X.S., Pan, Y.: Parametric optimization of pin-assisted-melt impregnation of glass fiber/polypropylene by taguchi method. J. Compos. Mater. 40(23), 2087- 2097(2006)

10. Varvani-Farahani, A.: Composite materials: characterization, fabrication and application-research challenges and directions. Appl. Compos. Mater. 17(2),63-67 (2000)

11. Gurvich, M.R., Dibenedetto, A.T., Pegoretti, A.: Evaluation of the statistical parameters of a Weibull distribution. J. Mater. Sci. 32(14), 3711-3716 (1997)

12. Turkovich, R., Erwin, L.: Fiber fracture in reinforced thermoplastic processing. Polym. Eng. Sci. 23(13), 743-749 (1983)

13. Astrom, B.T., Pipes, R.B.: A modeling approach to thermoplastic pultrusion. I: Formulation of models. Polym. Composite. 14(3), 173-183 (1993)

14. Astrom, B.T., Pipes, R.B.: A modeling approach to thermoplastic pultrusion. II: Verification of models. Polym. Composite. 14(3), 184-194 (1993)

15. Srinivasagupta, D., Potaraju, S., Kardos, J.L., Joseph, B.: Steady state and dynamic analysis of a bench-scale injected pultrusion process. Compos. Part A-Appl. S. 34(9), 835-846 (2003)

16. Yun, M.S., Lee, W.I.: Analysis of pulling force during pultrusion process of phenolic foam composites. Compos. Sci. Technol. 68(1), 140-146 (2008)

17. Durin, A., Micheli, P.D., Ville, J., Inceoglu, F., Valette, R., Vergnes, B.: A matricial approach of fibre breakage in twin-screw extrusion of glass fibres reinforced thermoplastics. Compos. Part A-Appl. S. 48, 47-56 (2013)

18. R'Mili, M., Bouchaour, T., Merle, P.: Estimation of Weibull parameters from loose-bundle tests. Compos. 
Sci. Technol. 56(7), 831-834 (1996)

19. Chen, C.: Mathematical modeling for the pultrusion of polymethyl methacrylate based composites. J. Appl. Polym. Sci. 123(4), 2228-2233 (1996)

20. Chen, C., Chen, I.: The unidirectional glass fiber reinforced furfuryl alcohol for pultrusion. II. correlation of processing parameters for optimizing the process. J. Appl. Polym. Sci. 119(3), 1788-1796 (2011)

21. Gaymans, R.J., Wevers, E.: Impregnation of a glass fibre roving with a polypropylene melt in a pin assisted process. Compos. Part A-Appl. S. 29(5-6), 663-670 (1998) 